\title{
THE TOXICITY OF THE HYDROLYSIS AND BREAKDOWN PRODUCTS OF MALATHION TO THE FATHEAD MINNOW (PIMEPHALES PROMELAS, RAFINESQUE)*
}

\author{
Michael E. Bender \\ Department of Environmental Health, University of Michigan, Ann Arbor, Michigan 48104
}

(Received 14 January 1969)

\begin{abstract}
Malathion undergoes hydrolysis in aqueous solutions; the products derived from the reaction are dependent upon the $\mathrm{pH}$ of the medium. It was the purpose of this investigation to evaluate the toxicity of the products of malathion hydrolysis in the fathead minnow, Pimephales promelas. The studies were conducted by toxicity bioassays of $96 \mathrm{hr}$ duration and continuous exposure tests of $\mathbf{1 4}$ day duration. The results of the experiments demonstrated the following: (1) the basic hydrolysis product, diethyl fumarate, was more toxic than malathion to the test species; (2) a pronounced synergistic effect was demonstrated between malathion and its two basic hydrolysis products; and (3) continuous exposure decreased the $T L m$ concentration of malathion and its basic hydrolysis products.
\end{abstract}

\section{INTRODUCTION}

THE USE of synthetic organic compounds for the control of insect pest species has created several problems in the aquatic environment. These include exposure of nontarget species to lethal and sub-lethal amounts of insecticides and their by-products, the concentration of these compounds in aquatic animals to levels injurious to the organism or its predators, and deleterious effects on the taste and odor of water and food species.

It has been generally accepted (RUDD, 1964 and JoHNSON, 1968) that the chlorinated hydrocarbons present a greater danger to non-target animals than do the organic phosphate pesticides, because of their greater acute toxicity and the persistence of their residues. There are, however, two basic fallacies in accepting, a priori, the view that the phosphate pesticides are less dangerous for wide use than the chlorinated hydrocarbon compounds. These are: (1) the effect of hydrogen ion concentration on the halflife of the phosphates; and (2) the production of other toxic compounds during the breakdown of the phosphate compounds.

Weiss and GAKSTATTER (1965) have shown that at a pH of below 7, malathion does not hydrolyse after prolonged periods. The work of MOUNT and STEPHAN (1967) on the effect of ten-month malathion exposures on the reproduction and growth of fathead minnows was in accordance with these results. Although their studies were not conducted at a pH where malathion could resist hydrolysis for prolonged periods, they maintained concentrations by using a continuous flow system. Their results demonstrated that continuous exposure at $1 / 45$ of the $96 \mathrm{hr}$ median tolerance limit concentration will not affect growth or reproduction of the fathead minnow.

The above studies do not, however, demonstrate all of the possible consequences arising from the use of malathion in the environment. For example, the two most neglected aspects of pesticide toxicity investigations on aquatic animals have been:

\footnotetext{
* These investigations were supported by USPHS Research Grant ES-00016-02 to Rutgers University.
} 
(1) the toxicity of the breakdown products of the parent compound has not been determined; and (2) the determination of the toxicity of the mixtures of these breakdown products has not been made. The toxicity of sevin, a carbamate insecticide, and its hydrolytic product, 1-naphthal, to marine animals has been investigated by STEWART et al. (1967) but aside from this, little attention has been given to the problem of toxicity changes associated with pesticide breakdown.

When malathion undergoes hydrolysis in aqueous solutions, the products derived from the reaction are dependent upon the $\mathrm{pH}$ of the medium. MUHLMANN and SCHRODER (1957) have studied the hydrolysis products of malathion and found that in basic solutions the primary products are diethyl fumarate and dimethyl phophorodithioic acid (DMPTA). In acid solutions the products are dimethyl phophorothionic acid and 2-mercaptodiethyl succinate.

It was the purpose of this investigation to use the fathead minnow to establish the toxicity of the hydrolysis products of malathion and other postulated by products (i.e., products arising from further hydrolysis products). Detection of possible synergistic and/or antagonistic effects between the parent compound and its basic hydrolysis products made up the other major area of the investigation.

\section{METHODS}

The fathead minnow, Pimephales promelas, was chosen as the experimental animal for the bioassay investigations. After collection in the field, all fish were allowed to acclimate to laboratory conditions for at least one week before use. During this period they were fed frozen brine shrimp.

The $96 \mathrm{hr}$ bioassay test employed to measure toxicity was performed as outlined in Standard Methods (1960) at a temperature of $17^{\circ} \mathrm{C}$. Ten fish with a combined weight of no more than $15 \mathrm{~g}$ were placed in 51 . tap water which had been aerated to remove chlorine and filtered through carbon to remove trace organic compounds. All compounds tested were added under the surface of the water with graduated pipettes from acetone stock solutions. The test vessels were checked for mortality at the end of $24 \mathrm{hr}$ of the test period, and dead fish were removed whenever observed. The $96 \mathrm{hr}$ static tests were used to determine the median tolerance limit $(T L m)$ of all compounds investigated. These compounds included the known hydrolysis products of malathion and some of its possible metabolites. A minimum of three experiments using at least 180 fish were used for each of these compounds. During the test the chemical parameters were: oxygen 7-8 mg/1, pH 7.0-7.3, alkalinity $50-70 \mathrm{mg} / 1$, dissolved solids $60-100 \mathrm{mg} / \mathrm{l}$, and iron less than $0.1 \mathrm{mg} / \mathrm{l}$.

As mentioned earlier, one of the most neglected aspects of insecticide toxicity investigations on aquatic animals has been the failure of investigators to determine the possible effect of breakdown products on the toxicity of the parent compound. The breakdown of malathion in basic aqueous solutions to diethyl fumarate and dimethyl phosphorodithioic acid creates an opportunity for synergistic and/or antagonistic effects. In this study these effects were investigated using a $3 \times 3 \times 3$ factorial design and $24 \mathrm{hr}$ bioassay tests of the same format as described for the $96 \mathrm{hr}$ tests.

In single dose static tests after the application of the chemical to the test container, its concentration may be reduced by hydrolysis, adsorption, or by uptake by the fish. In order to establish whether these factors influenced the results obtained with the 
major compounds of interest in this study, continuous exposure tests of 14 day duration were conducted on malathion and its basic hydrolysis products. Malathion solutions were metered into the test containers at a rate of $61 /$ day while the flow rates for diethyl fumarate and dimethyl phosphorodithioic acid solutions were 3 and 91/day, respectively. These rates were shown by chemical determinations on the effluents from the test chambers to be necessary to maintain the levels used in the tests.

All chemicals used in the experiments were either purified in the laboratory by column chromatography or were of analytical grade. The known hydrolysis products (diethyl fumarate, dimethyl phosphorothionic acid, dimethyl phosphorodithioic acid and 2-mercaptodiethyl succinate) were supplied by the American Cyanamid Company, other postulated breakdown products were obtained from various manufacturers in either reagent or analytical grade.

\section{RESULTS AND DISCUSSION}

\section{Static $96 \mathrm{hr}$ TLm test}

The median tolerance limits for malathion, its hydrolysis products, and the probable metabolites tested, as established by the static bioassay procedure, are listed in TABLE 1 .

There has been considerable debate on methods of establishing "safe" concentrations of toxic chemicals for aquatic life (TARzWELL, 1962). Most workers in the field have emphasized the necessity of determining these "safe" levels by continuous exposure studies in which the growth and reproductive success of the aquatic animals of interest are considered (MouNT, 1967). The labor and time required for performing such tests are, however, quite extensive, as shown by the work of MouNT and STEPHAN (1967). Their expenditure can only be justified if the life expectancy of the chemical of interest and the extent to which the chemical occurs in the aquatic environment are known.

The concentrations of each of the hydrolysis and postulated breakdown products which could be produced from the complete breakdown of $13.5 \mathrm{mg} / \mathrm{l}$ of malathion are also shown in TABLE 1. Without a study of the effects of these estimated concentrations on aquatic animals no sound statements as to their possible significance can be made.

However, some "educated" estimates are possible, based on the work which has been done on the establishment of safety factors. BURDICK (1967) mentions the use of a 0.1 factor times the $96 \mathrm{hr} T L \mathrm{Lm}$ as a means of calculating the "safe" concentration; while EDWARDS and BROWN (1966) have suggested that a "safe level" may be about 0.4 of the $96 \mathrm{hr} T L m$. HART, et al. (1945) have given the following formula for estimation of a "safe" concentration:

$$
C=0.348 \mathrm{hr} T L m /(24 \mathrm{hr} / 48 \mathrm{hr} T L m)^{2} .
$$

The proportions of the estimated safe concentration calculated by each of these methods, based on the amount of the compound produced by complete hydrolysis of a $T L m$ concentration of malathion are shown in TABLE 1 . Based on these estimates of "safe" concentrations, two compounds, maleic acid and diethyl fumarate, exceed the limits for safety established by all methods of estimation, while diethyl maleate and dimethyl phosphate exceed the limit in two cases. More than half of the compounds tested exceed the "safe" concentrations when using a factor of 0.1 . 


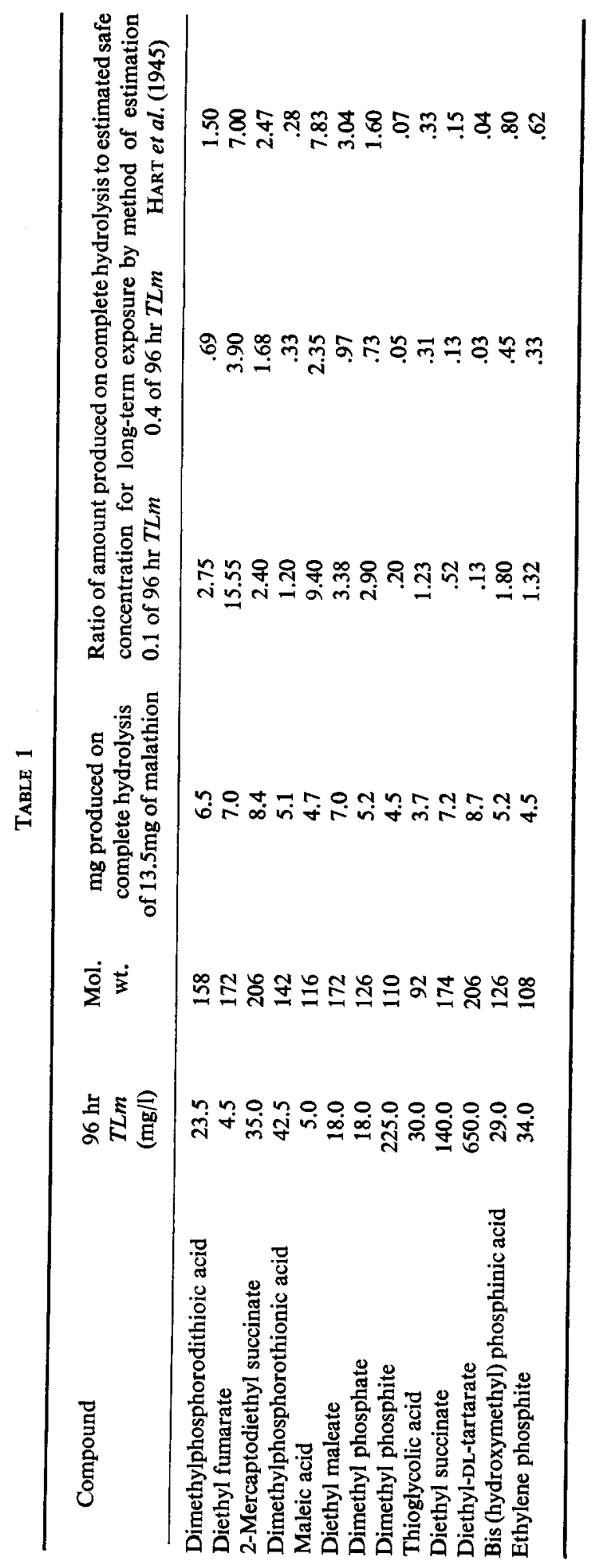


From these considerations, it is evident that a complex system of toxic effects could arise from the hydrolysis or metabolism of malathion. The extent to which this occurs in the natural environment is dependent upon the actual quantities of the compounds produced and their subsequent ability to resist further breakdown.

\section{Toxicity curves}

Toxicity response curves for malathion and its basic hydrolysis products were also established with the $96 \mathrm{hr}$ static bioassay tests. Response to the toxicant concentration was measured by recording the per cent mortality at each level in $96 \mathrm{hr}$. Ninety-five per cent confidence limits were calculated on the data, and these results are graphically presented in FIG. 1.

The most striking feature of the response curves is the difference in their range of response. The range of response expressed in concentration units within which $0-100$ per cent mortality occurred for diethyl fumarate was only $4 \mathrm{mg} / \mathrm{l}$, while DMPTA had a range of $7 \mathrm{mg} / 1$ and malathion, $16 \mathrm{mg} / \mathrm{l}$.

The wide range of response shown by the fathead minnow when exposed to malathion is indicative of the ability of the animal's systems to function within fairly broad limits of stress. In support of this conclusion WeIss (1958) has shown that fish are able to function until brain acetyl-cholinesterase levels are lowered from 60 to 30 per cent of normal. The narrow range of response observed for diethyl fumarate suggests a mode of action different from that of malathion. This conclusion is also supported by observations on the fishes' behavior on exposure to the two compounds. Death from phosphate insecticide poisoning can be characterized by erratic swimming patterns, muscle tetanus and flexed opercula. Fish succumbing to diethyl fumarate poisoning showed none of these symptoms and were observed simply dying.

\section{Continuous flow tests}

FIGURE 2 shows the change in TLm values for malathion and its basic hydrolysis products as a function of time. All of the tests showed a decrease in the TLm concentration compared to the standard $96 \mathrm{hr}$ test.

The significance of these lower $T L m$ values must not only take into account the stability which the compound shows in nature but also the possible quantities which could result from malathion hydrolysis. These longer term tests are undoubtedly of little practical significance for diethyl fumarate since at the $\mathrm{pH}$ of its production ( $\mathrm{pH} \mathrm{8}$ ) it remains stable for only 4 days. The situation for DMPTA is of similar nature in that only small amounts, in relation to its $T L m$ concentration, could be produced upon complete hydrolysis of $13.5 \mathrm{mg} / 1$ malathion. The 14 day test for malathion can be considered realistic on the basis of its stability at $\mathrm{pH}$ levels of six and seven. However, its importance in nature would depend on quantity and frequency of application.

\section{Synergism}

The effects of the basic hydrolysis products of malathion on the toxicity of resulting mixtures were measured using a $3 \times 3 \times 3$ factorial design with three replications. The concentrations used were chosen with the aid of the toxicity response curves and preliminary trial runs.

Per cent mortalities were transformed by the arc sine method (DAvies, 1963). The analysis of variance shown on TABLE 2 was performed after this transformation. Since

\section{C $\mathbf{W}$}



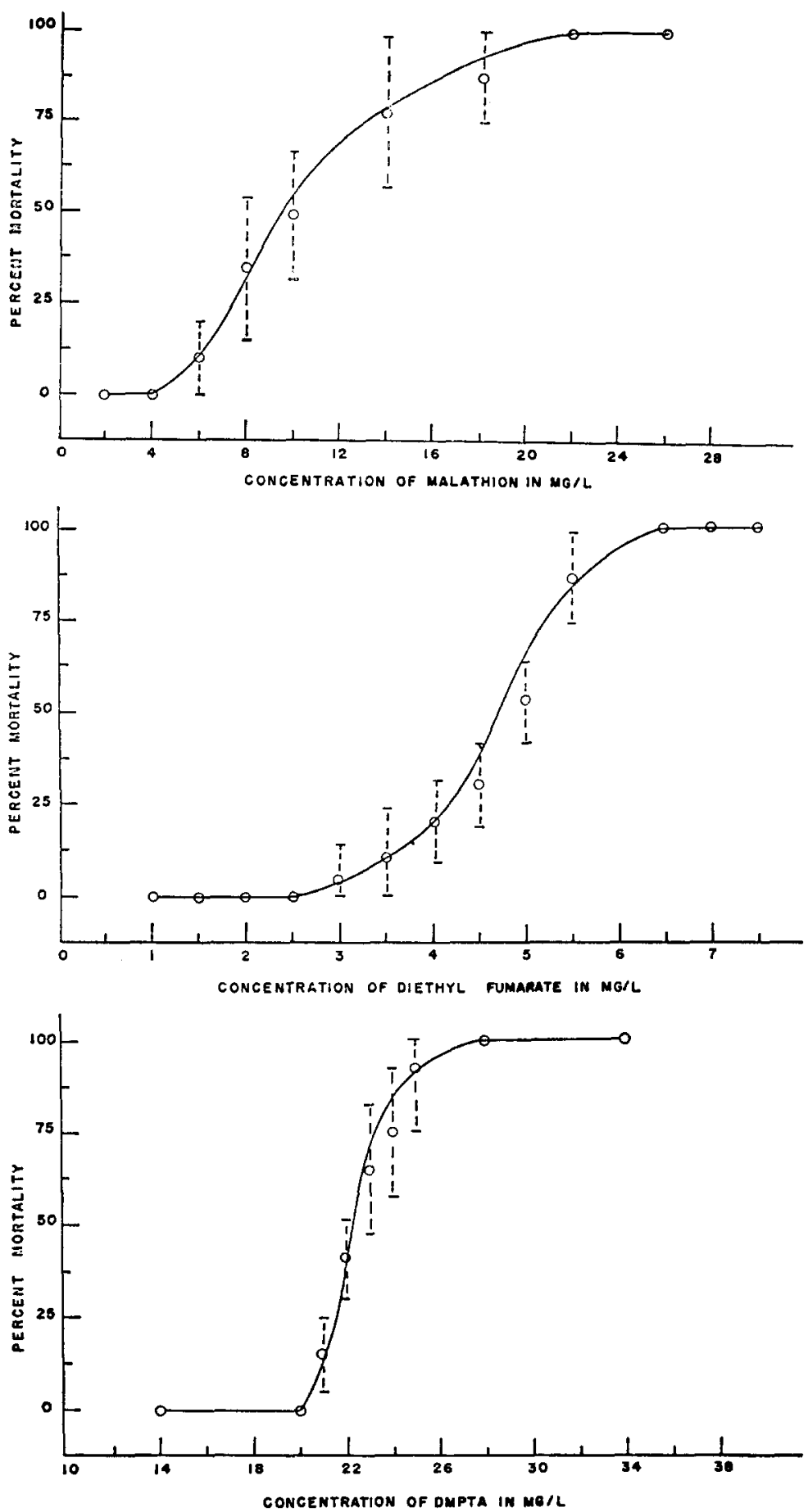

FIG. 1. Toxicity response curves for malathion and its basic hydrolysis products, confidence limits at 95 per cent level. 

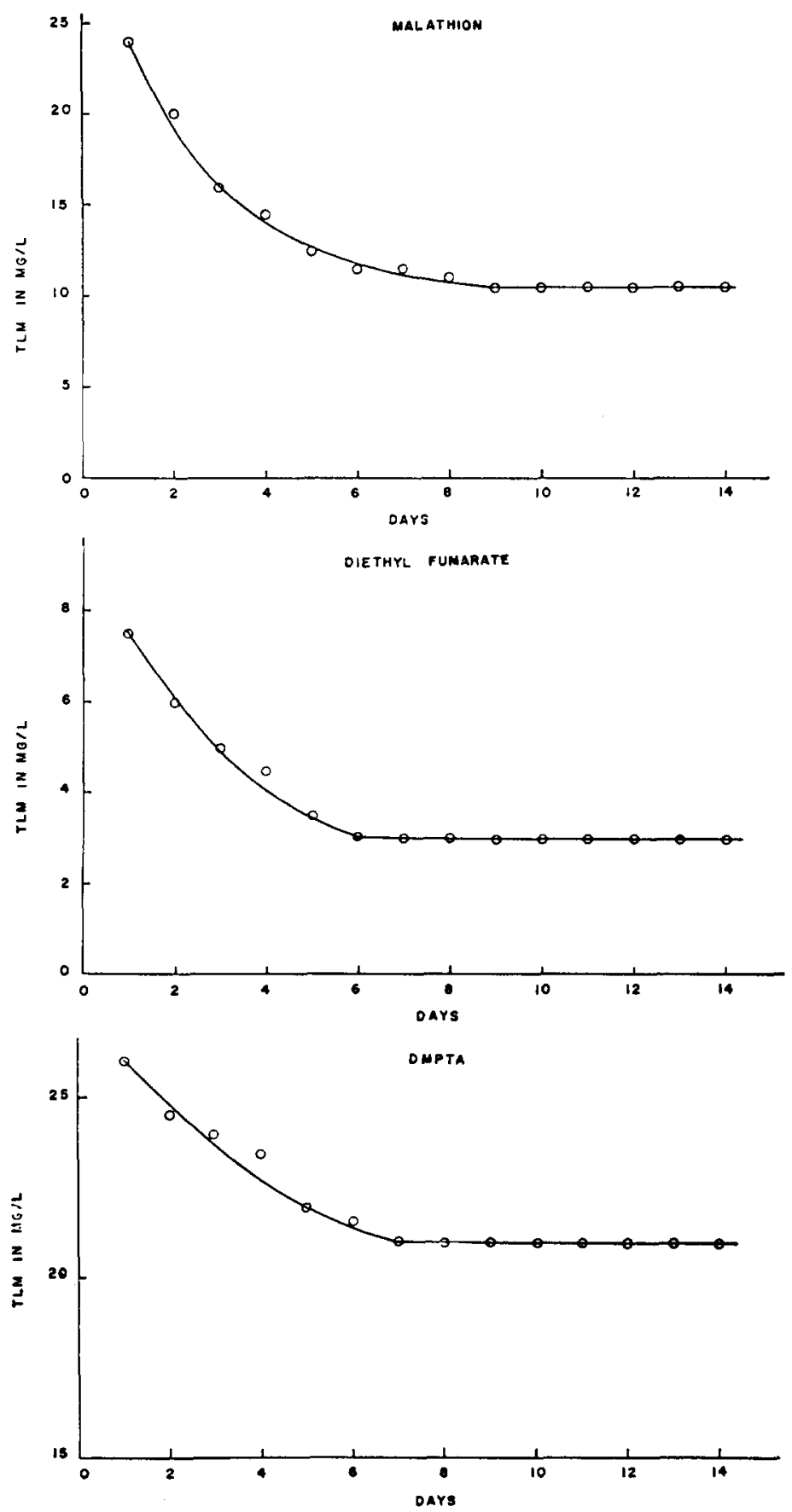

FIG. 2. $T L m$ concentration of malathion and its basic hydrolysis products, plotted as a function of time, from continuous exposure tests. 
the major interest was only in the possible interaction, it would not be logical to discuss the significance of the main effects. Hence, only the $F$ ratios for the interactions are of interest; all proved to be significant at the 0.01 level.

FIGURE 3 shows the two way interactions. All three of the two way interactions appear similar with respect to the large increases in toxicity, which were produced when two of the compounds were present in solution. In other words, the toxicities of the compounds were not additive, but an accelerating or synergistic effect was produced when they were both present. The use of both higher levels did not prove very important in showing this effect, except possibly to point out that the higher of the two levels was more toxic.

TABLE 2. SYNERGISM EXPERIMENT: MALATHION AND ITS BREAKDOWN PRODUCTS-ANALYSIS OF VARIANCE

\begin{tabular}{lrrrr}
\hline Source of variation & Sum of squares & D.F. & Mean square & $F$ \\
\hline Malathion & 14.9799 & 2 & 7.4899 & 505.8208 \\
DMPTA & 3.9884 & 2 & 1.9942 & 134.6738 \\
Diethyl Fumarate & 2.9260 & 2 & 1.4630 & 98.8010 \\
M D DMPTA & 1.5981 & 4 & 0.3995 & 26.9817 \\
M D.F, & 1.4251 & 4 & 0.3563 & 24.0602 \\
DMPTA $\times$ D.F. & 1.0103 & 4 & 0.2526 & 17.0565 \\
M $\times$ DMPTA $\times$ D.F. & 0.6614 & 8 & 0.0827 & 5.5832 \\
Error (within cell) & 0.7996 & 54 & 0.0148 & \\
& & & & \\
\hline
\end{tabular}

To complicate the picture even further, the three way interaction proved significant. Concise graphic resentation of this interaction is presented in FIG. 4. One can visualize this interaction as follows (e.g., FIG. 4b); (1) the toxicity due to diethyl fumarate at one level is determined over three levels of malathion; (2) also separately at the three levels of DMPTA; (3) this procedure is then followed for the other two levels of diethyl fumarate; and (4) the process is then repeated using each compound in step number one. This is necessary to visualize the entire problem. However, the three way interaction can be visualized, for example, as shown in FIG. 4c, by observing the change in response at one level of diethyl fumarate plotted at the separate levels of malathion produced by varying the levels of DMPTA.

Based on calculations of the concentrations of hydrolysis products theoretically possible from a $T L m$ concentration of malathion, as performed earlier, it appears that only diethyl fumarate could be produced in significant quantities to produce the synergistic effect in nature. Dimethyl phosphorothioic acid could only be present at a level of $6.5 \mathrm{mg} / \mathrm{l}$ on complete hydrolysis of malathion, and in preliminary experiments no synergism was observed using a level of $5 \mathrm{mg} / \mathrm{l}$.

\section{SUMMARY AND CONCLUSIONS}

The static $96 \mathrm{hr}$ bioassays performed on malathion and its postulated hydrolysis products brought to light several interesting points. The first of these concerns the acute toxicity of the known basic hydrolysis product diethyl fumarate, whose $96 \mathrm{hr}$ $T L m$ was found to be $4.5 \mathrm{mg} / \mathrm{l}$. This quantity of diethyl fumarate would be produced from the hydrolysis of 64 per cent of the $T L m$ concentration of malathion for the fathead minnow. 


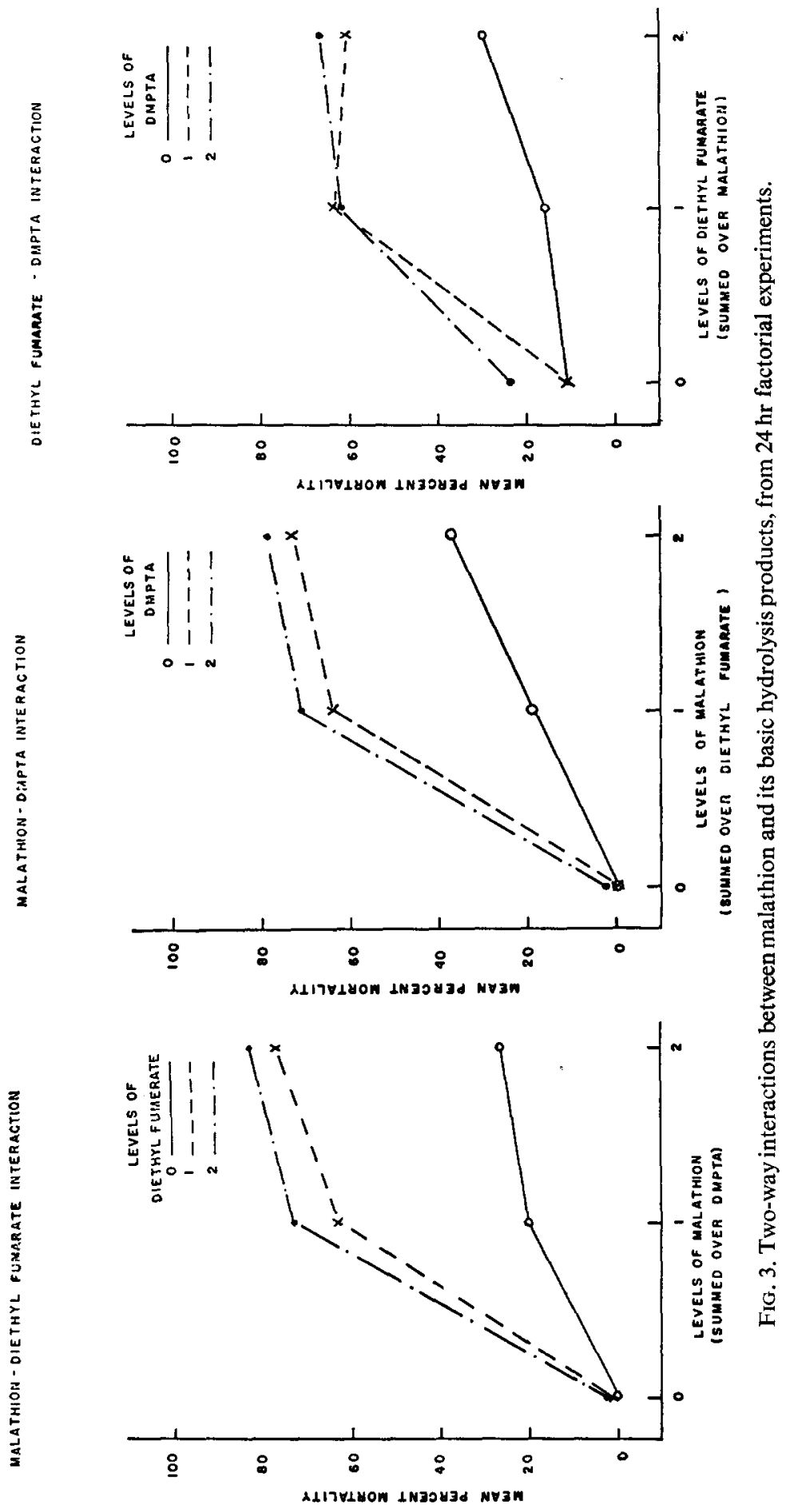



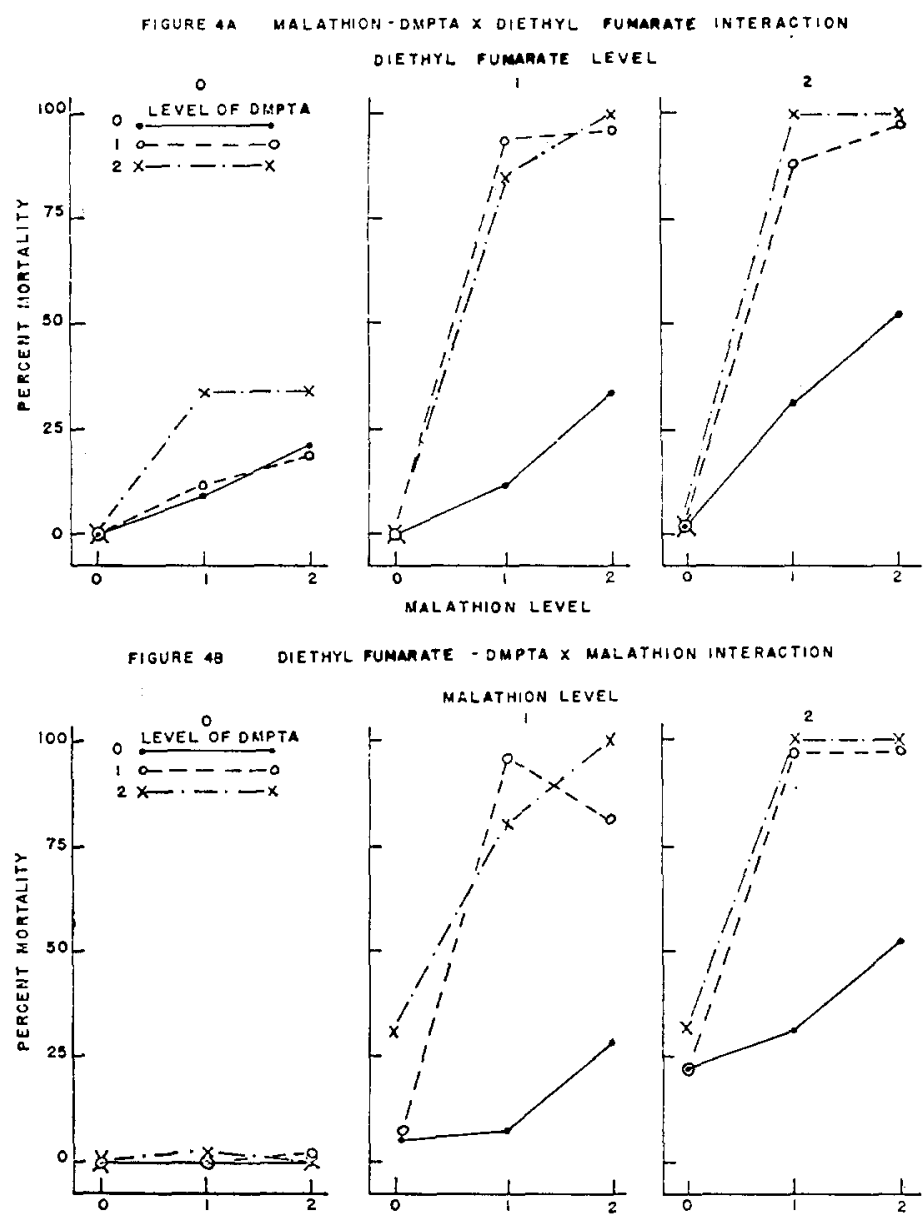

DIETHYL FUMARATE LEVEL

FIGUREAC MALATHION-DIETHYI. FUMATATE X DMPTA INTERACTION

DMPTA LEVEL
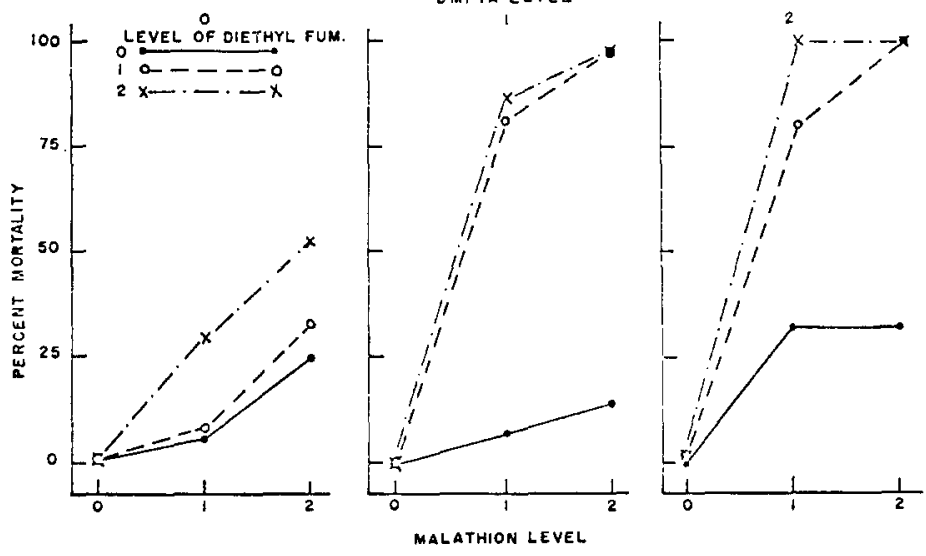

FIG. 4. Three-way interactions between malathion and its basic hydrolysis products, from $24 \mathrm{hr}$ factorial experiments. 
Calculations of safe concentrations of toxic materials for aquatic animals were made based on three proposed methods. These estimates showed that at least three of the proposed hydrolysis products, diethyl maleate, maleic acid and diethyl fumarate could be produced in quantities sufficient to cause long term damage, assuming of course that they either persist long enough or were continuously added to the environment. These results point out the need for experimental investigations to determine the actual amounts of the compounds produced, their persistence in the aquatic environment, and if concentrations present warrant investigation, their possible long term effect. The need to determine the possible effect of these hydrolysis products on other more sensitive species is also indicated. Moreover, from the data presented, it is evident that a toxicity investigation on a parent compound alone is hardly an adequate basis for the licensing or recommendation of particular compound for widespread use.

Existence of a pronounced synergistic effect between malathion and its basic hydrolysis products was shown. The possible implications of this effect in field applications of malathion can not be overemphasized, for it is quite simple to postulate field conditions which could result in synergism playing significant role in mortality. The following examples will serve to illustrate this point. A difference of a day or two in the application time of malathion on two adjacent areas could result in a condition in which a considerable quantity of the breakdown product along with a substantial quantity of the parent compound could be washed by rainfall into a common water source. Even more likely to occur is the application of malathion over a 2-3 day period, a time in which considerable quantities of both compounds could result and be carried into a common stream. Another possible situation would be its application to two adjacent drainage areas differing slightly in $\mathrm{pH}$, one basic the other neutral or acid; if then at some future time these two water sources were to converge, synergism could very easily result. Even in a single body of water the daily $\mathrm{pH}$ fiuctuations could conceivably be responsible for producing synergistic conditions. The rapid production of diethyl fumarate could occur during the period when $\mathrm{pH}$ is high, its rate of production being reduced after the active photosynthetic period, leaving enough malathion to produce the effect. The synergistic effect of malathion and diethyl fumarate could affect a wide range of target and non-target animals, and the assessment of this possibility would seem to play an essential role in establishing the effect of malathion contamination in the environment.

\section{REFERENCES}

BuRDicr G. E. (1967) Use of bioassays in determining levels of toxic wastes harmful to aquatic organisms. Am. Fish. Soc. Special Publication No. 4, 3-12.

DAvies O. L. (1963) Editor: The Design and Analysis of Industrial Experiments $636 \mathrm{pp}$. Hafner, New York.

Edwards R. W. and Brown V. M. (1966) Pollution and fisheries. In Institute of Sewage Purification, Annual Conference Vol. 1, pp. 49-55.

HaRT W. B., Doudoroff P. and GreendanK J. (1945) The Evaluation of Industrial Wastes, Chemicals and Other Substances to Freshwater Fishes $317 \mathrm{pp}$. Waste Control Laboratory of the Atlantic Refining Co.

Johnson D. W. (1968) Pesticides and fishes-a review of selected literature. Trans. Am. Fish. Soc. 97, $398-424$.

Mount D. I. (1967) Considerations for acceptable concentrations of pesticides for fish production. Am. Fish. Soc. Special Publication No. 4, 3-6. 
MOUNT D. I. and STEPHAN C. E. (1967) A method for establishing acceptable toxicant limits for fishmalathion and the butoxyethanol ester of 2, 4-D. Trans. Am. Fish. Soc. 96, 185-193.

MUhLMAN V. R. and SchradER G. (1957) Hydrolyse der insektiziden phosphorsauresster. Z. Naturf. 12, 196-208.

Rudd R. L. (1964) Pesticides and the Living Landscape 320 p. Univ. of Wisc. Press, Madison.

Standard Methods for the Examination of Water and Waste Water (1960) 11 th Ed. 626 pp. Am. Publ Hlth Ass. New York.

Stewart N. E., Millemann R. E. and Greese W. P. (1967) Acute toxicity of the insecticide sevin and its hydrolytic product 1-naphthol to some marine organisms. Trans. Am. Fish. Soc. 96, 25-31.

TARZWELl C. M. (1962) Development of water quality criteria for aquatic life. J. Wat. Pollut. Control Fed. 34, 1178-1185.

WeIss C. M. (1958) The determination of chlorinesterase in the brain tissue of three species of fresh water fish and its inactivation in vivo. Ecology 39, 194-199.

Weiss C. M. and Gakstatter J. H. (1965) The decay of anticholinesterase activity of organic phosphorus insecticides on storage in waters of different pH. Proc. 2nd Int. Water Pollution Res. Conf., Tokyo, pp. 83-95. 